\title{
Size quantization in metal films
}

\author{
S.S. Nedorezov \\ Kharkov National Automobile-road University, 25 Petrovskogo Str., Kharkov 610002, Ukraine \\ E-mail:Eme-Tatyana@yandex.ru
}

Receive January 29, 2007

\begin{abstract}
Quantum size effect, predicted by I.M. Lifshits and A.M. Kosevich [Izv. Akad. Nauk SSSR, seriya fiz. 19, 395 (1955)], was investigates in a many works. In the basis of analysis of quantum size oscillations of thermodynamics and kinetic characteristics of metal films lies the quasiclassical quantization of component of momentum for isotropy model and quantization [S.S. Nedorezov, Zh. Eksp. Teor. Fiz. 51, 868 (1966) [Sov. Phys.-JETF 24, 578 (1967)]] of chord of constant-energy surface in the case of anisotropy energetic spectrum. In the given work the research of quantum size levels of energy of electrons in metal films is carried out by the method of J.M. Luttinger-W.Kohn. The exact conditions of size quantization are got.
\end{abstract}

PACS: 71.61.-r Electrical properties of specific thin films;

71.15.Nc Total energy and cohesive energy calculations;

73.21.Fg Quantum wells.

Keywords: band, chord, dispersion equation, oscillations.

1. The electronic energy spectrum of metallic film is determined by the Schrödinger equation of the electron in the periodic potential of crystal taking into account potential $U(z)$, created by the border surface of film. On the condition

$$
a<<l<<L,
$$

where $a$ - distance between atoms, $l-$ characteristic distance, on which the potential $U(z)$ changes substantially, $L$ - thickness of a film with normal along the axis of $z$, the decision of given equation can be found by the method of Luttinger-Kohn [1] and approximate potential $U(z)$ by the infinitely deep potential pit. A similar method was used by an author at the analysis of size-quantum levels of energy in semiconductor films [2].

We suppose the decision of the Schrödinger equation in a kind

$$
\Psi\left(\mathbf{x}_{\|}, z\right)=\sum_{s} \exp \left(\frac{i}{\hbar} \mathbf{p}_{\|} \mathbf{x}_{\|}\right) F_{S}(z) u_{s 0}\left(\mathbf{x}_{\|}, z\right),
$$

where $\mathbf{x}_{\|}=(x, y), \mathbf{p}_{\|}=\left(p_{x}, p_{y}\right), \hat{\mathbf{p}}=\left(\mathbf{p}_{\|},-i \hbar d / d z\right), s-$ labeling the bands, $u_{s 0}$ - the Bloch function at the bottom of band $E_{s}$ in a point $\mathbf{p}=0$. Here the functions $F_{s}(z)$ satisfy zero border condition and are the decision of the system of differential equations

$$
\begin{aligned}
& \left(E_{s}+\frac{p_{x}^{2}+p_{y}^{2}}{2 m}-\frac{\hbar^{2}}{2 m} \frac{d^{2}}{d z^{2}}\right) F_{S}(z)+ \\
& +\sum_{s^{\prime} \neq s} \frac{1}{m}\left(\hat{\mathbf{p}} \cdot \mathbf{K}_{s s^{\prime}}\right) F_{s^{\prime}}(z)=\varepsilon F_{S}(z),
\end{aligned}
$$

where

$$
\mathbf{K}_{s s^{\prime}}=\frac{(2 \pi \hbar)^{3}}{\Omega} \iiint_{\Omega} u_{s 0}^{*}\left(-i \hbar \frac{\partial}{\partial \mathbf{r}}\right) u_{s^{\prime} 0} d^{3} \mathbf{r}
$$

is the momentum matrix elements, $\Omega$ is the volume of the unit cell.

The given decision is written down in a kind

$$
F_{S}(z)=\sum_{j=1}^{2 N} A_{s j} \exp \left(\frac{i}{\hbar} p_{z j} z\right)
$$

where $N$ is number of the bands, $p_{z j}$ is roots of dispersion equation

$$
\begin{aligned}
& \operatorname{det} \|\left(E_{s}+\frac{1}{2 m} p_{\|}^{2}-\varepsilon+\frac{1}{2 m} p_{z j}^{2}\right) \delta_{s s^{\prime}}+ \\
& +\left(\mathbf{p}_{\|} \mathbf{K}_{\| s s^{\prime}}+p_{z j} K_{z s s^{\prime}}\right)\left(1-\delta_{s s^{\prime}}\right) \|=0,
\end{aligned}
$$

which can be by imaginaries. Amplitudes $A_{s j}$ are determined by the homogeneous system of algebraic equations 


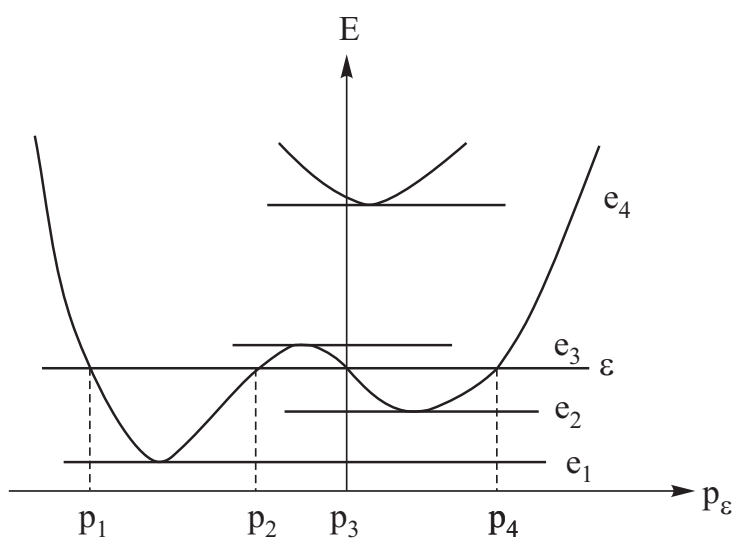

Fig. 1. The dependence of the energy bands $E_{1,2}\left(\mathbf{p}_{\|}, p_{z}\right)$ on component of the momentum $p_{z}$.

$$
\begin{aligned}
& \left(E_{s}+\frac{1}{2 m} p_{\|}^{2}-\varepsilon+\frac{1}{2 m} p_{z j}^{2}\right) A_{s j}+ \\
& +\sum_{s^{\prime} \neq s}^{N}\left(\mathbf{p}_{\|} \mathbf{K}_{\| s s^{\prime}}+p_{z j} K_{z s s^{\prime}}\right) A_{s^{\prime} j}=0 .
\end{aligned}
$$

The decision of equations (7) contains $2 N$ arbitrary constants, $s=1,2, \ldots, N ; j=1,2, \ldots, 2 N$.

Taking (5) into zero border condition, we get

$$
\sum_{j=1}^{2 N} B_{l j}=0, \quad l=1,2, \ldots, 2 N
$$

where

$$
B_{l j}=\left\{\begin{array}{l}
A_{l j}, 1 \leq l \leq N, \\
A_{l-N, j} \exp \left(\frac{i}{\hbar} p_{z j} L\right), N+1 \leq l \leq 2 N .
\end{array}\right\} .
$$

$$
\operatorname{det}|| B_{l j}||=0 \text {, }
$$

which determines the quantum size levels of energy (subbands) $\varepsilon_{n}\left(\mathbf{p}_{\|}\right)$.

2. We will consider a model of two bands more in detail. In this case energy bands $E_{1,2}\left(\mathbf{p}_{\|}, p_{z}\right)$ have a kind

$$
\begin{aligned}
& E_{1,2}\left(\mathbf{p}_{\|}, p_{z}\right)=\frac{E_{1}+E_{2}}{2}+\frac{p_{\|}^{2}+p_{z}^{2}}{2 m} \mp \\
& \mp \sqrt{\left(\frac{E_{2}-E_{1}}{2}\right)^{2}+\frac{1}{m}\left(\mathbf{K}_{\|} \mathbf{p}_{\|}+K p_{z}\right)^{2}},
\end{aligned}
$$

where $\mathbf{K}_{\|}=\mathbf{K}_{\| 1,2}, K=K_{z 1,2}$. From (10), supposing $N=2$, we get dispersion equation

$$
\begin{gathered}
\quad \sin \frac{L}{2 \hbar}\left(p_{2}-p_{1}\right) \sin \frac{L}{2 \hbar}\left(p_{4}-p_{3}\right)= \\
=W \sin \frac{L}{2 \hbar}\left(p_{3}-p_{2}\right) \sin \frac{L}{2 \hbar}\left(p_{4}-p_{1}\right),
\end{gathered}
$$

where $p_{j}$ is roots of equation

$$
E_{1,2}\left(\mathbf{p}_{\|}, p_{j}\right)=\varepsilon, \quad j=1,2,3,4
$$

$$
W=\frac{\left(\alpha_{4}-\alpha_{3}\right)\left(\alpha_{2}-\alpha_{1}\right)}{\left(\alpha_{4}-\alpha_{1}\right)\left(\alpha_{3}-\alpha_{2}\right)}, \alpha_{j}=\frac{2 m\left(\varepsilon-E_{1}\right)-p_{\|}^{2}-p_{j}^{2}}{\mathbf{K}_{\|} \mathbf{p}_{\|}+K p_{j}} \text {. }
$$

In the interval of energies $e_{2}<\varepsilon<e_{3}$ and $\varepsilon>e_{4}$ (see Fig. 1) all roots have the material value.

If $|W|<1$ from dispersion equation (13) the conditions of quantization follow

$$
p_{2}-p_{1}=\frac{2 \pi \hbar}{L}\left(n+\gamma_{1}\right), p_{4}-p_{3}=\frac{2 \pi \hbar}{L}\left(n+\gamma_{2}\right),
$$

where

From (8) we get dispersion equation

$$
\begin{aligned}
& \gamma_{1}=\frac{1}{\pi} \operatorname{arctg} \frac{W \sin (L / 2 \hbar)\left(p_{4}-p_{1}\right) \sin (L / 2 \hbar)\left(p_{3}-p_{1}\right)}{\sin (L / 2 \hbar)\left(p_{4}-p_{3}\right)+W \sin (L / 2 \hbar)\left(p_{4}-p_{1}\right) \cos (L / 2 \hbar)\left(p_{3}-p_{1}\right)}, \\
& \gamma_{2}=\frac{1}{\pi} \operatorname{arctg} \frac{W \sin (L / 2 \hbar)\left(p_{3}-p_{2}\right) \sin (L / 2 \hbar)\left(p_{3}-p_{1}\right)}{\sin (L / 2 \hbar)\left(p_{2}-p_{1}\right)-W \sin (L / 2 \hbar)\left(p_{3}-p_{2}\right) \cos (L / 2 \hbar)\left(p_{3}-p_{1}\right)} .
\end{aligned}
$$

If $|W|>1$ we have

$$
p_{3}-p_{2}=\frac{2 \pi \hbar}{L}\left(n+\gamma_{3}\right), p_{4}-p_{1}=\frac{2 \pi \hbar}{L}\left(n+\gamma_{4}\right),
$$

where

$$
\begin{aligned}
& \gamma_{3}=\frac{1}{\pi} \operatorname{arctg} \frac{\sin (L / 2 \hbar)\left(p_{2}-p_{1}\right) \sin (L / 2 \hbar)\left(p_{4}-p_{1}\right)}{W \sin (L / 2 \hbar)\left(p_{4}-p_{1}\right)+\sin (L / 2 \hbar)\left(p_{2}-p_{1}\right) \cos (L / 2 \hbar)\left(p_{4}-p_{2}\right)}, \\
& \gamma_{4}=\frac{1}{\pi} \operatorname{arctg} \frac{\sin (L / 2 \hbar)\left(p_{2}-p_{1}\right) \sin (L / 2 \hbar)\left(p_{3}-p_{1}\right)}{-W \sin (L / 2 \hbar)\left(p_{3}-p_{2}\right)+\sin (L / 2 \hbar)\left(p_{2}-p_{1}\right) \cos (L / 2 \hbar)\left(p_{3}-p_{1}\right)} .
\end{aligned}
$$

The within the framework of considered model of two bands the got the conditions of quantization are exact and are written down in a kind comfortable for the analysis of quantum size oscillations of thermodynamics and kinetic 
quantities. At $n>>1$ these conditions of quantization within sizes $\gamma$ coincide with the quasiclassical condition of size quantization, got in [3] $]^{*}$.

In the interval of energies $e_{3}<\varepsilon<e_{4}$ (see Fig. 1) roots $p_{2}$ and $p_{3}$ are complex numbers

$$
p_{2}=p_{0}-i q, p_{3}=p_{0}+i q .
$$

In this case the condition of quantization is determined by formulas

$$
p_{4}-p_{1}=\frac{2 \pi \hbar}{L}\left(n+\gamma_{0}\right)
$$

where

$$
\begin{gathered}
\gamma_{0}=\frac{1}{\pi} \operatorname{arctg} \frac{\operatorname{ch}(L q / \hbar)-\cos (L / \hbar)\left(p_{0}-p_{1}\right)}{2 W_{0} \operatorname{sh}(L q / \hbar)+\sin (L / \hbar)\left(p_{0}-p_{1}\right)}, \\
W_{0}=\frac{\beta^{2}+\left(\alpha_{4}-\alpha\right)\left(\alpha_{1}-\alpha\right)}{2 \beta\left(\alpha_{4}-\alpha_{1}\right)} .
\end{gathered}
$$

Here, meaning $\mathbf{P}_{0}=\left(\mathbf{p}_{\|}, p_{0}\right)$, we have

$$
\begin{gathered}
\alpha=\frac{\left(\mathbf{K}_{12} \mathbf{P}_{0}\right)\left(2 m\left(\varepsilon-E_{1}\right)-\mathbf{P}_{0}^{2}+q^{2}\right)}{\left(\mathbf{K}_{12} \mathbf{P}_{0}\right)^{2}+(K q)^{2}}, \\
\beta=\frac{q\left[2 p_{0}\left(\mathbf{K}_{12} \mathbf{P}_{0}\right)+K\left(2 m\left(\varepsilon-E_{1}\right)-\mathbf{P}_{0}^{2}+q^{2}\right)\right]}{\left(\mathbf{K}_{12} \mathbf{P}_{0}\right)^{2}+(K q)^{2}} .
\end{gathered}
$$

In the interval of energies $e_{1}<\varepsilon<e_{2}$ the roots $p_{3}$ and $p_{4}$ are complex numbers. In this case the condition of quantization is determined by formulas similar to the formulas (17), (18).

3. The quantum oscillations of thermodynamics [4] and kinetic [5] quantities in dependence on thickness of film are due to size quantization of energy of conductive electrons. This was discovered experimentally on films of bismuth [6] and antimony [7]. In obedience to the general theory of oscillatory effects [4], a period and amplitude $C$ of quantum size oscillations depend on the extreme values of function $n_{e}(\varepsilon)=n\left(p_{x e}, p_{y e}, \varepsilon\right)$ and its derivative $d n_{e} / d \varepsilon$ at $\varepsilon=\varepsilon_{F}$, where $\varepsilon_{F}$ is the Fermi level, $\partial n_{e} / \partial p_{x}=0, \partial n_{e} / \partial p_{y}=0$. From the received higher the conditions of quantization we find the function

$$
n\left(p_{x}, p_{y}, \varepsilon\right)=\frac{L}{2 \pi \hbar} D\left(\mathbf{p}_{\|}, \varepsilon\right)-\gamma\left(\mathbf{p}_{\|}, \varepsilon, L\right),
$$

satisfying to equality $\varepsilon_{n}\left(\mathbf{p}_{\|}\right)=\varepsilon$. Here $D$ is a chord of constant-energy surface, a function $\gamma$ is limited $(|\gamma|<1)$ and substantially $(\sim L)$ changes with the change $\varepsilon$.

Taking $\frac{L}{2 \pi \hbar} D_{e}>1$ into account, from (19) for the period of the oscillations we get

$$
\Delta L=\frac{2 \pi \hbar}{D_{\text {extr }}} .
$$

Here $D_{\text {extr }}$ is the extreme chord of the Fermi surface (FS). The amplitude $C$ of considered oscillations is proportional $\Psi\left[2 \pi^{2} k T /(\Delta \varepsilon)\right]$, where

$$
\Delta \varepsilon=\frac{2 \pi \hbar}{L\left|D_{\text {extr }}^{\prime}(\varepsilon)-\frac{2 \pi \hbar}{L} \frac{\partial}{\partial \varepsilon} \gamma\left(p_{x e}, p_{y e}, \varepsilon, L\right)\right|},
$$

$\Psi(x)=x / \operatorname{sh}(x), T$ is temperature, $k=1,2,3 \ldots$

At an edge of band it is $\varepsilon_{F} \rightarrow e_{1}, e_{2}, e_{3}, e_{4}$ (see Fig. 1), $|W|<<1$ or $|W|>>1$, then from (21) follows

$$
\Delta_{0} \varepsilon=\frac{2 \pi \hbar}{L\left|D_{\mathrm{extr}}^{\prime}(\varepsilon)\right|}
$$

that corresponds to the results got from the quasiclassical condition of the quantization (see $[3,8]$ ).

With leaving from the edge of band approach (22) becomes inapplicable. The quantity $\Delta \varepsilon$ is calculated on a formula (21), where along with $D^{\prime}\left(\varepsilon_{F}\right)$ it is necessary to take into account $\frac{\partial}{\partial \varepsilon} \gamma\left(p_{x e}, p_{y e}, \varepsilon_{F}, L\right)$. The periods of quantum size oscillations are determined (see (20)) by the extreme chords SF. Thus, the presence of the second band a near Fermi level, not changing a period, substantially changes amplitude of quantum size oscillations. The value $\Delta \varepsilon$ can turn out more the value $\Delta_{0} \varepsilon$ that will result in more slow decrease of oscillations with the increase of temperature.

Experimental investigation $[6,7,9,10]$ of quantum size effects in semimetal films show clearly the presence of periodic oscillations of physical quantities not only at low temperatures, but also at temperatures $T \sim 100 \mathrm{~K}$. It does not consent with estimation $\Psi\left(2 \pi^{2} k T /\left(\Delta_{0} \varepsilon\right)\right.$ of the amplitude of oscillations, got from quasiclassical quantization. It is necessary to take into account the closeness of two bands in the energy spectrum of electrons in bismuth. It is possible to do within the framework of foregoing theory.

The exact conditions of size quantization got in this work allow to find the quantum sizes levels of energy of electrons of conductivity in a film of metal. It can serve by the basis of research of the effects conditioned discreteness of energy spectrum of electrons in a film.

More than 50 years passed from the prediction [4] of quantum size effects. The interest to these effects does not weaken (see, for example, [11]).

\footnotetext{
* Work [3] is executed by an author under the guidance and at the direct participation of I.M. Lifshits.
} 
1. J.M. Luttinger and W. Kohn, Phys. Rev. 97, 869 (1955).

2. S.S. Nedorezov, Fiz. Tverd. Tela 12, 2269 (1970) [Sov. Phys.-Solid State 12, 1814 (1971)].

3. S.S. Nedorezov, Zh. Eksp. Teor. Fiz. 51, 868 (1966) [Sov. Phys.-JETF 24, 578 (1967)].

4. I.M. Lifshits and A.M. Kosevich, Izv. Akad. Nauk SSSR, seriya fiz. 19, 395 (1955).

5. V.B. Sandomirskiū, Zh. Eksp. Teor. Fiz. 52, 158 (1967).

6. Yu.F. Ogrin, V.N. Lutski1̌, and M.I. Elinson, Pis'ma Zh. Eksp.Teor. Fiz. 3, 114 (1966).
7. Yu.F. Komnik and E.I. Bukhshtab, Pis'ma Zh. Eksp.Teor. Fiz. 6, 536 (1967).

8. I.O. Kulik, Pis'ma Zh. Eksp.Teor. Fiz. 6, 652 (1967).

9. Yu.F. Ogrin, V.N. Lutskiı̌, M.U. Arifova, V.I. Kovalev, V.B. Sandomirski1̌, and M.I. Elinson, Zh. Eksp. Teor. Fiz. 53,1218 (1967).

10. H.A. Combet and J.Y. Le Traon, Solid State Commun. 6, 85 (1968).

11. E. Ogando, N. Zabala, E.V. Chulkov and M.J. Puska. Phys. Rev. B69, 153410 (2004). 